\title{
ON NON-PROJECTIVE BLOCK COMPONENTS OF LEFSCHETZ CHARACTERS FOR SPORADIC GEOMETRIES
}

\author{
R. PHILIP GRIZZARD
}

\begin{abstract}
This work examines the possible projectivity of 2-modular block parts of non-projective Lefschetz characters over 2-local geometries of several sporadic groups. Previously known results on $M_{12}, J_{2}$, and $H S$ are mentioned for completeness. The main new results are on the sporadic groups $\mathrm{Suz}, \mathrm{Co}_{3}, \mathrm{Ru}, \mathrm{O}^{\prime} \mathrm{N}$, and $\mathrm{He}$. For each group, the Lefschetz character is calculated, and its 2-modular block parts are examined for projectivity. In each case it is confirmed that a non-principal block part contains a non-projective summand. The case of $O^{\prime} N$ is additionally found to have a non-projective summand in its principal block part. Nineteen of the sporadic groups (including many previously known cases) are categorized into three classes based on projectivity properties of their Lefschetz characters.
\end{abstract}

\section{INTRODUCTION}

Of the 26 sporadic groups, 11 have 2-local geometries with mod 2 projective Lefschetz modules, and most of these have been calculated previously RSY90. Of the remaining 15 with non-projective Lefschetz modules, the Lefschetz characters in terms of irreducibles already have been calculated for three of these groups. On the other hand, there are 7 of the remaining 12 sporadics whose 2 -modular irreducibles are not yet known. Thus there remain 5 sporadic groups with non-projective Lefschetz modules whose 2modular irreducibles are known [Bre99], but whose Lefschetz characters in characteristic 2 have not yet been discussed in the literature.

In this work, we calculate the Lefschetz characters and their 2-modular block decompositions for these five sporadic groups, namely $\mathrm{Suz}, \mathrm{O}^{\prime} \mathrm{N}, \mathrm{He}$, $\mathrm{Co}_{3}$, and $R u$. We find that the Lefschetz character for $O^{\prime} N$ has a nonprojective constituent in its principal block part, while the principal block parts for the other four groups each has the same character as a projective module.

This paper is a version of the author's Ph.D. thesis at the University of Illinois at Chicago.

Date: September 7, 2018.

1991 Mathematics Subject Classification. Primary: 20D08, 20F32, 20C34; Secondary: 20J05, 20C40.

The author was partially supported by NSA grant H 98230-05-1-0075. 


\section{Notation and Assumed Results}

Definitions. The (reduced) Lefschetz module of a complex $\Delta$ with action by a group $G$ is the virtual $\mathbb{Z} G$-module given by the alternating sum of the chain groups (cf. [RSY90, p. 281]). Its degree term is the (reduced) Euler characteristic: $\tilde{\chi}(\Delta):=\sum_{i=-1}^{\operatorname{dim} \Delta}(-1)^{i} \operatorname{dim} C_{i}(\Delta)$. We may reduce this module $\bmod 2$, and then analyze it using the 2-modular representation theory of $G$.

Definition 2.1 (Lefschetz character). The Lefschetz character of the natural 2-local geometry 1 of $G$ is the sequence of Euler characteristic values of the subgeometries fixed by representatives $g$ of each conjugacy class of $G$ : $\operatorname{Tr}(g, \widetilde{L})=\widetilde{\chi}\left(\Delta^{g}\right)$. We denote the Lefschetz character of $G$ by $\widetilde{\Lambda}_{G}$, or just $\widetilde{\Lambda}$ when the group is unambiguous.

Let $B$ be a particular ${ }^{2}$-modular block for $G$. Then we define $\widetilde{\Lambda}_{B}^{G}$ to be the component of $\widetilde{\Lambda}_{G}$ coming from Brauer characters in $B$.

Definition 2.2 (Projective). We define $\Phi\left(\varphi_{i}\right)$ as the character of the projective cover $P\left(\varphi_{i}\right)$ of the 2-modular irreducible Brauer character $\varphi_{i}$. We say a module has projective character if its character can be written as a sum of $\Phi\left(\varphi_{i}\right)$.

Definition 2.3 (Virtual projective). A virtual projective module is a $\mathbb{Z}$ linear combination of projective indecomposables $P(\varphi)$. We say a virtual module has v-projective character if the module has the same character as that of a virtual projective module, i.e. if its character equals some $\mathbb{Z}$-linear combination of $\Phi\left(\varphi_{i}\right)$. When describing the character itself with this property, we say the character is v-projective. We emphasize that if a module has v-projective character, that does not necessarily imply that the module is a virtual projective module.

Tests for projectivity. We use two standard properties of projective modules below. For each of these, the contrapositive presents a quick check for failure of a Lefschetz module to be projective. The second condition is stronger.

Lemma 2.4 (The $p$-test). If $\widetilde{L}(\Delta)$ is projective, then $|G|_{p}$ divides $\widetilde{\chi}(\Delta)$.

Lemma 2.5 (The vanishing test). [Fei82, p.148] For the module $\widetilde{L}(\Delta)$, its Lefschetz character $\widetilde{\Lambda}$ is v-projective if and only if $\widetilde{\Lambda}$ vanishes at the nontrivial 2-elements.

If a Lefschetz character passes the vanishing test, this indicates that the character is v-projective, i.e. can be expressed as a combination of $\Phi\left(\varphi_{i}\right)$.

\section{Some REMARKS ON THE RESUlts OF OUR STUDY}

\footnotetext{
1"2-local geometry" is defined in Benson-Smith [BS07, §7.2].
} 
Partition of the sporadics based on their Lefschetz characters. We define Class I groups as having Lefschetz modules that are projective. We will refer to each group in this class as a "Lefschetz Module Projective."

A Class II group is one whose Lefschetz module is non-projective, yet whose Lefschetz module restricted to the principal block part has v-projective character (perhaps zero). This is the case with three groups previously studied in the literature, and we will show this to be the case with four more sporadics. We will refer to a sporadic group in this class as a "Principal Block Part V-Projective."

Finally, a Class III group contains a non-projective summand in the principle block part of its Lefschetz module - and this part has no cohomology 2 since Benson-Smith established that the Lefschetz module is acyclic. Though there are standard constructions in the literature [CR94 of such modules, this work concretely exhibits (for the first time) an example of such a module naturally occurring in the context of $\widetilde{L}(\Delta)$ : in the O'Nan group. We will refer to a group in this class as a "Principal Block Part Non-Projective."

Other observations. In section 8.2, Benson and Smith [BS07] remark that reduced Lefschetz module appears always to involve an indecomposable in a non-principal block. After examining larger groups, we can reformulate their comment as follows.

Remark 3.1 (Non-principal block observation). Each sporadic group affording a non-projective Lefschetz module appears to have a non-projective part in a non-principal block.

This observation holds in each case we study, and we conjecture that it will hold for the remaining seven sporadics.

In addition, Smith Smi05 noticed a further pattern: the defect of the largest non-principal block part seems to equal the 2-power difference between $|\widetilde{\chi}(\Delta)|_{2}$ and $|G|_{2}$. Of the sporadic groups we study, only $O^{\prime} N$ fails to follow this further pattern 3

Remark 3.2. In these 15 sporadic cases where $\widetilde{L}(\Delta)$ is not projective, the vanishing test 2.5 guarantees that there will be at least one 2-element $b$ for which $\Delta^{b}$ is not contractible. We mention an experimental observation: this nonzero value for the Euler characteristic often matches the Euler characteristic value for a well-known non-contractible geometry. This hints that

\footnotetext{
${ }^{2}$ In particular, the variety of this principal block part must lie in the representation theoretic nucleus, as introduced by Benson-Carlson-Robinson [BCR90, p. 68] (see also Benson Ben02, prop. 1.1].

${ }^{3}$ For an approach to this pattern via vertices of modules, see Sawabe Saw06.
} 
perhaps our geometric structure $\Delta^{b}$ is homotopy equivalent to that wellknown geometry. We will suggest such possible geometries for seven groups in our study

\section{REVIEW OF KNOWN CASES}

We present the Lefschetz characters of $M_{12}, J_{2}$, and $H S$, and then proceed to display their 2-modular block decompositions.

The Mathieu group $M_{12}$. Benson-Wilkerson [BW95, p. 44] computed $\widetilde{\Lambda}_{M_{12}}=\chi_{14}+2 \chi_{15}$. These characters lie in the unique non-principal block. We express this as a vector with columns indexed by $M_{12}$ conjugacy classes: $\widetilde{\Lambda}_{M_{12}}=[\overbrace{496}^{2^{4} \cdot 31}, \frac{\mathbf{2 A}}{-\mathbf{4}}, \frac{\mathbf{2 B}}{\mathbf{0}},-8,-5,0,0,1,-1,0,0,0,1,1,1]$.

The vanishing test indicates that $\Delta^{2 A}$ is not contractible. From the viewpoint of Remark 3.2, the value -4 in $\widetilde{\Lambda}_{M_{12}}$ matches the degree of the Lefschetz module of the building $\Sigma$ of a subgroup $L_{2}(4)$ in the centralizer of a $2 \mathrm{~A}$ element. This suggests that $\Delta^{2 A}$ may be homotopy equivalent to this $\Sigma$. Finally, one concise way of expressing the Lefschetz character via possible v-projective and non-projective parts is: $\widetilde{\Lambda}_{M_{12}}=\Phi\left(\varphi_{6}\right)+\chi_{15}$.

The Janko group $J_{2}$. Benson and Smith [BS07, after Theorem 8.7.1] found $\widetilde{\Lambda}_{J_{2}}=\chi_{16}+\chi_{17}+\chi_{19}$, noting that these characters lie in the unique non-principal block. We calculate $\widetilde{\Lambda}_{J_{2}}=[\overbrace{736}^{2^{5} \cdot 23}, \frac{\mathbf{2 A}}{\mathbf{0}}, \frac{\mathbf{2 B}}{-\mathbf{4}}, 16,-5,0,1,1,-4,-4$, $0,-1,1,0,1,1,0,0,0,1,1]$. Again from the viewpoint of Remark 3.2, the value -4 at class $2 \mathrm{~B}$ matches the degree of $\widetilde{L}(\Sigma)$ for building $\Sigma$ of an $L_{2}(4)$ in $\mathrm{C}(2 \mathrm{~B})$, the centralizer of conjugacy class $2 \mathrm{~B}$. We can express the Lefschetz character as: $\widetilde{\Lambda}_{J_{2}}=\Phi\left(\varphi_{8}\right)+\chi_{16}$.

The Higman-Sims group $H S$. Klaus Lux calculated the Lefschetz character for $H S$ in a private communication to Stephen D. Smith:

$\widetilde{\Lambda}_{H S}=\overbrace{42624}^{2^{7} \cdot 333}, \frac{\mathbf{2 A}}{\mathbf{0}}, \frac{\mathbf{2 B}}{-\mathbf{1 6}},-36,0,0,0,24,-1,4,2,0,1,0,0,0,0,-1,-1,-1,0$, $-1,0,0]$. In the viewpoint of Remark 3.2, the value of -16 is the degree of $\widetilde{L}(\Sigma)$ for $\Sigma$ the building of $S p_{4}(2)$ in $\mathrm{C}(2 \mathrm{~B})$.

Block decomposition. In this case we have some contribution from both blocks. For the principal block, we find that $\widetilde{\Lambda}_{p r}^{H S}=2 \Phi\left(\varphi_{8}\right)$, so this is v-projective. The "closest" we could come to expressing the block 2 part as a v-projective character is $\widetilde{\Lambda}_{b 2}^{H S}=\Phi\left(\varphi_{6}\right)+\Phi\left(\varphi_{7}\right)+\Phi\left(\varphi_{9}\right)+\chi_{24}$.

We separate the Lefschetz character values according to the 2-modular blocks: $\widetilde{\Lambda}_{p r}^{H S}=[\overbrace{26624}^{2^{11} \cdot 13}, \frac{\mathbf{2 A}}{\mathbf{0}}, \frac{\mathbf{2 B}}{\mathbf{0}},-16,0,0,0,24,24,4,0,0,-4,0,0,0,0$,

\footnotetext{
${ }^{4}$ After a preprint version of this paper was circulated, Silvia Onofrei and John Maginnis [MO0] established many such homotopy equivalences.
} 
$0,4,4,0,-6,0,0]$ and $\widetilde{\Lambda}_{b 2}^{H S}=[\overbrace{16000}^{2^{7} \cdot 125}, \frac{\mathbf{2 A}}{\mathbf{0}}, \frac{\mathbf{2 B}}{-\mathbf{1 6}},-20,0,0,0,0,-25,0,2$, $0,5,0,0,0,0,-1,-5,-5,0,5,0,0]$. We see by the vanishing test that the non-projective part of $\widetilde{\Lambda}_{H S}$ is contained completely in the non-principal block, which is consistent with Remark 3.1 .

\section{The Suzuki Group}

We study the maximal subgroups of $S u z$ which contain a Sylow 2-group $\mathrm{CCN}^{+} 85$, p. 131]:

$$
\begin{aligned}
& H_{1} \cong 2_{-}^{1+6} \cdot U_{4}(2) \\
& H_{2} \cong 2^{2+8}:\left(A_{5} \times S_{3}\right), \text { and } \\
& H_{4} \cong 2^{4+6}: 3 A_{6}
\end{aligned}
$$

Geometry. The diagram for the natural geometry of $S u z$ is given in RonanSmith [RS80, p. 288] as:

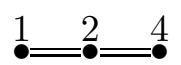

We proceed as in Benson-Smith [BS07, Def. 5.3.2]. The set $I:=\{1,2,4\}$ indexes the "simplex of subgroups" $\mathcal{H}_{I}$ of $S u z$. This simplex is determined by the $H_{i}$, with $H_{J}:=\bigcap_{i \in J} H_{i}$ for all nonempty subsets $J \subseteq I$. For simplicity, we will use the notation $H_{i j}$ (without braces) to represent the subgroup $H_{\{i, j\}}$, etc.

The Lefschetz character in this geometry is an alternating sum of the permutation representations on vertices (with stabilizers $H_{1}, H_{2}$, and $H_{4}$ ), edges (with stabilizers $H_{12}, H_{14}, H_{24}$ ), and 2-simplices with stabilizer $H_{124}$.

Inducing from subgroups. We calculate Lefschetz characters by inducing trivial representations through a chain of subgroups: from intersections of $H_{1}, H_{2}$, and $H_{4}$ up to the maximal subgroups themselves, and then up to the whole group 5

We then combine these in an alternating sum. Since $H_{1}, H_{2}$, and $H_{4}$ are maximal subgroups of $S u z$, the permutation characters on their cosets are readily available in GAP Gro99]6 The permutation characters for the intersections of the maximal subgroups, however, are not contained in GAP. Instead, selected residues (i.e. links of simplices) described by the diagram tell us which characters of the intersections are needed in the calculation. We will investigate $\operatorname{Res}\left(H_{1}\right)$, in order to induce the trivial characters of $H_{12}$, $H_{14}$, and $H_{124}$ up to $H_{1}$. Then we use $\operatorname{Res}\left(H_{4}\right)$ to induce from $H_{24}$ up to $H_{4}$. GAP can then induce the resulting characters from $H_{1}$ and $H_{4}$ up to $S u z$, as well as the trivial characters for the maximal subgroups $H_{1}, H_{2}, H_{4}$ up to $S u z$.

\footnotetext{
${ }^{5}$ This process is described and several examples are given in section 4 of [RSY90].

${ }^{6}$ I am grateful to Professor Robert A. Wilson of Queen Mary University of London for showing me the step-by-step details of this process.
} 
Using the diagram, we find that inducing the trivial character of $H_{12}$ up to $H_{1}$ will be the inflation of the trivial character of $2^{4}: A_{5}$ induced up to $U_{4}(2)$. The Atlas gives this character to be $1 a+6 a+20 a$. So we have (by the transitivity of induction):

$$
[1 a]_{H_{12}} \uparrow^{S u z}=\left([1 a]_{H_{12}} \uparrow^{H_{1}}\right)_{H_{1}} \uparrow^{S u z}=[1 a+6 a+20 a]_{H_{1}} \uparrow^{S u z} .
$$

The diagram also tells us that $[1 a]_{H_{14}} \uparrow H_{1}$ is the inflation of the trivial character of $2\left(A_{4} \times A_{4}\right) \cdot 2$ induced up to $U_{4}(2)$, which is $1 a+20 a+24 a$, so:

$$
[1 a]_{H_{14}} \uparrow^{S u z}=\left([1 a]_{H_{14}} \uparrow^{H_{1}}\right)_{H_{1}} \uparrow^{S u z}=[1 a+20 a+24 a]_{H_{1}} \uparrow^{S u z} .
$$

Similarly $[1 a]_{H_{124}} \uparrow^{H_{12}}$ is the inflation of $[1 a]_{A_{4}} \uparrow^{A_{5}}=1 a+4 a$, giving us:

$$
[1 a]_{H_{124}} \uparrow^{S u z}=\left(\left([1 a]_{H_{124}} \uparrow^{H_{12}}\right)_{H_{12}} \uparrow^{H_{1}}\right)_{H_{1}} \uparrow^{S u z}=\left([1 a+4 a]_{H_{12}} \uparrow^{H_{1}}\right)_{H_{1}} \uparrow^{S u z} .
$$

There is a mild complication here, in that GAP does not contain the character tables of maximal subgroups such as $2^{4}: A_{5}$ of $U_{4}(2)$, so it is not set up to do the induction $[4 a]_{H_{12}} \uparrow^{H_{1}}$. But this can be calculated using the viewpoint of Smith [Smi90]. We display the result in the style of the Atlas:

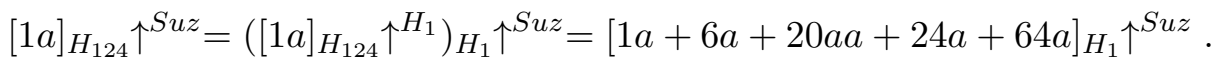

$\operatorname{Res}\left(H_{1}\right)$ has taken care of all but one of the intersections of maximal subgroups whose trivial characters we need to induce up to Suz. The last one needed is $H_{24}$, which we examine in $\operatorname{Res}\left(H_{2}\right)$. We have:

$$
[1 a]_{H_{24}} \uparrow^{S u z}=\left([1 a]_{H_{24}} \uparrow^{H_{2}}\right)_{H_{2}} \uparrow^{S u z}=[1 a+4 a]_{H_{2}} \uparrow^{S u z} .
$$

We are now ready to calculate the Lefschetz character of $S u z$ as an alternating sum, with the sign at a simplex $\sigma$ given by $(-1)^{\operatorname{dim} \sigma}$. To summarize our above findings:

$$
\begin{aligned}
{[1 a]_{H_{124}} \uparrow^{S u z} } & =[1 a+6 a+20 a a+24 a+64 a]_{H_{1}} \uparrow^{S u z} \\
-[1 a]_{H_{12}} \uparrow^{S u z} & =-[1 a+6 a+20 a]_{H_{1} \uparrow} \text { Suz } \\
-[1 a]_{H_{14}} \uparrow^{S u z} & =-[1 a+20 a+24 a]_{H_{1}} \uparrow^{S u z} \\
{[1 a]_{H_{1}} \uparrow^{S u z} } & =[1 a]_{H_{1} \uparrow} \uparrow^{S u z} \\
-[1 a]_{H_{24}} \uparrow^{S u z} & =-[1 a+4 a]_{H_{2}} \uparrow^{S u z} \\
{[1 a]_{H_{2}} \uparrow^{S u z} } & =[1 a]_{H_{2}} \uparrow^{S u z} \\
{[1 a]_{H_{4}} \uparrow^{S u z} } & =[1 a]_{H_{4}} \uparrow^{S u z}
\end{aligned}
$$

We can do some quick cancellation to simplify:

$$
\widetilde{\Lambda}_{S u z}=[64 a]_{H_{1} \uparrow} \uparrow^{S u z}-[4 a]_{H_{2}} \uparrow^{S u z}+[1 a]_{H_{4}} \uparrow^{S u z}-1 .
$$

The result is: $\widetilde{\Lambda}_{S u z}=[4189184,0,-64,3968,-352,-73,0,0,0,8,-16,9,0$, $0,0,0,-1,-1,0,0,0,2,2,0,1,-1,0,0,0,-1,0,-1,-1,-1,2,2,3,0,0,0,-1$, $-1,0]$. This is the Lefschetz character in vector form, i.e., the columns are indexed by the conjugacy classes. 
Block decomposition. As a sum of irreducible complex characters, we get $\widetilde{\Lambda}_{\text {Suz }}=[0,0,0,0,0,0,0,0,0,0,0,0,0,0,0,0,0,0,0,0,0,0,0,2,1,1,1$, $0,1,0,1,1,0,0,2,2,1,2,2,2,2,2,3]$. This is the Lefschetz character in scalar product form, i.e., the columns are indexed by the irreducible complex characters of Suz.

We use these coefficients of irreducible characters along with the decomposition matrix to decompose $\widetilde{\Lambda}_{S u z}$ into v-projective and non-projective parts. We find that $\widetilde{\Lambda}_{p r}^{S u z}=\Phi\left(\varphi_{13}\right)+\Phi\left(\varphi_{14}\right)$, a v-projective character.

For block 2 (of defect 3 ), the "closest" we can come to decomposing $\widetilde{\Lambda}_{b 2}^{S u z}$ into v-projectives is: $\widetilde{\Lambda}_{b 2}^{S u z}=\Phi\left(\varphi_{15}\right)+\Phi\left(\varphi_{16}\right)+\chi_{43}$. Hence the block 2 part of the Lefschetz character of $S u z$ is non-projective, verifying our observation in Remark 3.1. Suz is categorized into Class II. Furthermore, the non-principal block part has defect 3 , which matches the 2-power difference between $|G|_{2}=$ $2^{13}$ and $|\widetilde{\chi}(\Delta)|_{2}=\left|\widetilde{\Lambda}_{S u z}\right|_{2}=2^{10}$, continuing the pattern noticed by Smith Smi05.

We summarize our findings with the following theorem.

Theorem 5.1. $\widetilde{\Lambda}_{p r}^{\text {Suz }}=\Phi\left(\varphi_{13}\right)+\Phi\left(\varphi_{14}\right)$. Thus Suz is a Class II "Principal Block Part V-Projective" group.

The Lefschetz character values separated into blocks. The vectors of Lefschetz character values (indexed by the conjugacy classes) given just by characters in each respective block are:

$\widetilde{\Lambda}_{p r}^{S u z}=\left[2834432, \frac{\mathbf{2 A}}{\mathbf{0}}, \frac{\mathbf{2 B}}{\mathbf{0}}, 512,-352,-64,0,0,0,0,32,32,0,0,0,0,0,-8,0\right.$, $0,0,-4,-4,0,0,-4,0,0,0,0,0,3,3,0,-4,-4,2,0,0,0,1,1,0]$ and $\widetilde{\Lambda}_{b 2}^{S u z}=$ $[1354752, \mathbf{0}, \mathbf{- 6 4}, 3456,0,-9,0,0,0,8,-48,-23,0,0,0,0,-1,7,0,0,0,6$, $6,0,1,3,0,0,0,-1,0,-4,-4,-1,6,6,1,0,0,0,-2,-2,0]$. Here we have used bold face for the values at elements of order 2 in order to make clear the results of the vanishing test 2.5, From the viewpoint of Remark 3.2, this value of -64 is also the degree of $\widetilde{L}(\Sigma)$ for the building $\Sigma$ of $L_{3}(4)$ in $\mathrm{C}(2 \mathrm{~B})$, suggesting a possibly homotopy equivalence.

\section{The O’Nan group}

For our remaining four groups starting with $O^{\prime} N$, we present a somewhat more abbreviated study with fewer details than with Suz. The maximal subgroups of interest are $\left[\mathrm{CCN}^{+} 85\right.$, p. 132]:

$$
\begin{aligned}
& H_{1} \cong 4_{2} \cdot L_{3}(4): 2_{1}, \text { and } \\
& H_{3} \cong 4^{3} \cdot L_{3}(2) .
\end{aligned}
$$

With only two maximal subgroups, the process of obtaining the Lefschetz character is much simpler. There is no need for a diagram, and we have only one intersection to find: $H_{13}$. We can induce the trivial character of $H_{13}$ via:

$$
[1 a]_{H_{13} \uparrow} \overbrace{}^{\prime} N=\left([1 a]_{H_{13}} \uparrow^{H_{3}}\right)_{H_{3}} \uparrow^{\prime} N=[1 a+6 a]_{H_{3}} \text { }^{\prime} N
$$


One easy cancellation leads to the final calculation:

$$
\widetilde{\Lambda}_{O^{\prime} N}=[1 a]_{H_{1} \uparrow O^{\prime} N}-[6 a]_{H_{3} \uparrow} O^{\prime} N-1 .
$$

Using GAP, we obtain the Lefschetz character (negating to obtain positive degree) $\widetilde{\Lambda}_{O^{\prime} N}=\overbrace{254294272}^{2^{8} \cdot 993337}, \mathbf{\mathbf { 2 A }} \mathbf{8 9 6 0},-44,0,0,-8,-4,-48,-13,0,0,0,1,0,0$, $1,1,0,0,0,0,1,1,1,0,0,0,0,1,1]$.

The principal block. We might expect that the principal block part has v-projective character as it did in Suz. But we find:

$$
\widetilde{\Lambda}_{p r}^{O^{\prime} N}=\overbrace{147299328}^{2^{10} \cdot 143847}, \frac{\mathbf{2 A}}{\mathbf{7 1 6 8}},-2736,0,0,708,-32,2388,344,0,0,28,176,
$$
$0,-28,-156,-156,0,0,0,0,80,80,80,0,0,0,0,-520,-520]$.

Notice that $\left|O^{\prime} N\right|_{2}=2^{9}$ does indeed divide $\left|\widetilde{\Lambda}_{p r}^{O^{\prime} N}\right|$, so the $p$-test 2.4 passes.

However, the vanishing test 2.5 fails, and so $\widetilde{\Lambda}_{p r}^{O^{\prime} N}$ is actually not projective. Indeed this block part is in some sense very far from being v-projective, as the "closest" expression (in terms of minimizing the coefficients of nonprojective components) we can find is:

$$
\begin{aligned}
\widetilde{\Lambda}_{p r}^{O^{\prime} N} & =20 \Phi\left(\varphi_{3}\right)+12 \Phi\left(\varphi_{7}\right)+16 \Phi\left(\varphi_{8}\right) \\
& +2 \chi_{8}+2 \chi_{9}+18 \chi_{11}+4 \chi_{12}+4 \chi_{13}+4 \chi_{14}+2 \chi_{15} \\
& +8 \chi_{18}+6 \chi_{19}+12 \chi_{20}+18 \chi_{21}+18 \chi_{22}+14 \chi_{25} .
\end{aligned}
$$

Block 2-defect 3. We compute $\widetilde{\Lambda}_{b 2}^{O^{\prime} N}=\overbrace{1386240}^{2^{8} .5415}, \frac{\mathbf{2 A}}{\mathbf{1 7 9 2}}, 1140,0,0,60,28$, $324,-12,0,0,-28,-24,0,28,60,60,0,0,0,0,0,0,0,0,0,0,0,-$ $18,-18]$. This block part is also non-projective, and we can write $\widetilde{\Lambda}_{b 2}^{O^{\prime} N}=$ $8 \Phi\left(\varphi_{2}\right)+4 \Phi\left(\varphi_{4}\right)+8 \Phi\left(\varphi_{5}\right)+14 \chi_{7}$.

The defect 0 blocks. The Lefschetz character of $O^{\prime} N$ has several constituents from blocks of defect 0 , which are of course projective: $\widetilde{\Lambda}_{b 3}^{O^{\prime} N}=$ $97 \varphi_{9}, \widetilde{\Lambda}_{b 4}^{O^{\prime} N}=97 \varphi_{10}, \widetilde{\Lambda}_{b 5}^{O^{\prime} N}=115 \varphi_{11}, \widetilde{\Lambda}_{b 6}^{O^{\prime} N}=115 \varphi_{12}, \widetilde{\Lambda}_{b 7}^{O^{\prime} N}=115 \varphi_{13}$. These do not seem very illuminating for our study.

Theorem 6.1. By the vanishing test 2.5, $\widetilde{\Lambda}_{p r}^{O^{\prime} N}$ is non-projective. Thus $O^{\prime} N$ is categorized into Class III-it is a "Principal Block Part Non-Projective" sporadic group.

Observations of unusual behavior. The principal block part of the Lefschetz character of $O^{\prime} N$ contains a non-projective summand, making $O^{\prime} N$ the charter member of Class III. This discovery for O'Nan provides the first "natural" example in the literature on sporadic geometries of such a principal block part with no cohomology.

The principal block part of $O^{\prime} N$ is the only block part we find in our study to pass the $p$-test 2.4, yet fail the vanishing test 2.5. This may be related to $O^{\prime} N$ being the only group in our study with only one conjugacy 
class of order 2. Furthermore, the value of $\widetilde{\Lambda}_{O^{\prime} N}$ at that involution is 8960 . This is an unfamiliar Euler characteristic value, and indeed $O^{\prime} N$ is the only group for which we do not make a homotopy equivalence conjecture as in Remark 3.2,

Finally, note that the largest non-principal block of $O^{\prime} N$ has defect 3 but the 2-power difference between $|\widetilde{\Lambda}|_{2}$ and $|G|_{2}$ is only 1 . This is the only example we study that violates the pattern observed by Smith Smi05.

\section{The Held group}

Our next group is the Held group He. The relevant maximal 2-local subgroups are $\left[\mathrm{CCN}^{+} 85\right.$, p. 131]:

$$
\begin{aligned}
& H_{1} \cong 2_{+}^{1+6} \cdot L_{3}(2), \\
& H_{6 a} \cong 2^{6}: 3 \cdot S_{6}, \text { and } \\
& H_{6 b} \cong 2^{6}: 3 \cdot S_{6} .
\end{aligned}
$$

The diagram is given in Ronan-Smith [RS80, p. 288] as:

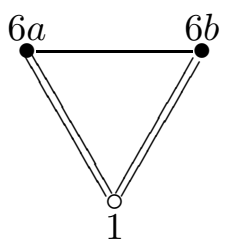

We obtain the subgroups

$$
H_{1,6 a}=H_{1,6 b}=S_{3} 2^{2} 2_{+}^{1+6}, H_{1,6 a, 6 b}=\frac{\frac{2}{2^{2}}}{2_{+}^{1+6}} \text {, and } H_{6 a, 6 b}=\frac{3\left(S_{4} \times 2\right)}{2^{6}} .
$$

Using previously described techniques, we find 7

$$
\widetilde{\Lambda}_{H e}=[8 a]_{H_{1} \uparrow}{ }^{H e}-[5 d+9 b]_{H_{6 a}} \uparrow^{H e}+[1 a]_{H_{6 b}} \uparrow^{H e}-1 .
$$

With columns indexed by the conjugacy classes of $H e$, we calculate:

$$
\begin{aligned}
& \widetilde{\Lambda}_{H e}=[\overbrace{1120384}^{2^{7} \cdot 8753}, \frac{\mathbf{2 A}}{-\mathbf{6 4}}, \frac{\mathbf{2 B}}{\mathbf{0}},-197,-8,-8,0,0,9,-1,0,-1,-1,-1,6,6,0,1,1, \\
& 0,-1,-1,0,0,3,-1,-1,-1,-1,-1,-1,-1,-1] .
\end{aligned}
$$

Block decompositions. $\widetilde{\Lambda}_{p r}^{H e}=\overbrace{760832}^{2^{10} .743}, \frac{\mathbf{2 A}}{\mathbf{0}}, \frac{\mathbf{2 B}}{\mathbf{0}}, 152,-40,0,0,0,-68,0,0$, $16,16,58,-12,-12,0,0,0,0,0,0,0,0,-8,14,14,-2,-2,-5,-5,0,0]$. Using the 2-modular table, we can express this as $\widetilde{\Lambda}_{p r}^{H e}=\Phi\left(\varphi_{10}\right)+\Phi\left(\varphi_{11}\right)+\Phi\left(\varphi_{12}\right)$. For block 2 (of defect 3), we have $\widetilde{\Lambda}_{b 2}^{H e}=\overbrace{58496}^{2^{7} \cdot 457}, \frac{\mathbf{2 A}}{-\mathbf{6 4}}, \frac{\mathbf{2 B}}{\mathbf{0}},-13,32,-8,0,0,21$, $-1,0,-17,-17,39,18,18,0,1,1,0,-1,-1,0,0,-3,-1,-1,-6,-6,4,4,-1,-1]$. This fails tests 2.4 and 2.5, so we use the decomposition matrix to see how "close" this block part is to being v-projective: $\widetilde{\Lambda}_{b 2}^{H e}=3 \Phi\left(\varphi_{14}\right)+\chi_{15}$. From

\footnotetext{
${ }^{7}$ I am grateful to Silvia Onofrei and John Maginnis for correcting an error at this point in my original calculation.
} 
the viewpoint of Remark 3.2 , this value of -64 is also the degree of $\widetilde{L}(\Sigma)$ for the building $\Sigma$ of $L_{3}(4)$ in $\mathrm{C}(2 \mathrm{~B})$. The parts in blocks 3 and 4 must be projective, since they have defect 0 . We have $\widetilde{\Lambda}_{b 3}^{H e}=7 \varphi_{15}$, and $\widetilde{\Lambda}_{b 4}^{H e}=7 \varphi_{16}$.

Theorem 7.1. $\widetilde{\Lambda}_{p r}^{H e}=\Phi\left(\varphi_{10}\right)+\Phi\left(\varphi_{11}\right)+\Phi\left(\varphi_{12}\right)$. Hence He is a Class II "Principal Block Part V-Projective" group.

\section{The Conway group $\mathrm{Co}_{3}$}

The maximal subgroups we examine are $\left[\mathrm{CCN}^{+} 85\right.$, p. 134]:

$$
\begin{aligned}
& H_{1} \cong 2^{\cdot S p_{6}}(2), \\
& H_{2} \cong 2^{2} \cdot\left[2^{7} \cdot 3^{2}\right] \cdot S_{3}, \text { and } \\
& H_{4} \cong 2^{4} \cdot A_{8} .
\end{aligned}
$$

The diagram for the natural geometry [BS07, §8.13] is:

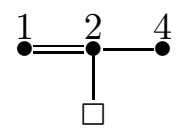

This gives us the needed intersections $H_{12}=2^{2} \cdot\left[2^{6}\right]:\left(S_{3} \times S_{3}\right), H_{14}=$ $2.2^{6}: L_{3}(2), H_{124}=2.2^{6}: S_{4}$, and $H_{24}=2^{4} .2^{4}:\left(S_{3} \times S_{3}\right)$. These lead us to:

$$
\widetilde{\Lambda}_{C o_{3}}=[216 a+280 b]_{H_{1} \uparrow} \uparrow^{C o_{3}}-[14 a+20 a]_{H_{4} \uparrow C o_{3}}+[1 a]_{H_{2} \uparrow C o_{3}}-1 \text {. }
$$

Now we use GAP to obtain $\widetilde{\Lambda}_{C_{0}}=[\overbrace{50378624}^{2^{7} \cdot 393583}, \frac{\mathbf{2 A}}{\mathbf{0}}, \frac{\mathbf{2 B}}{-\mathbf{4 9 6}},-2080,-784$, $125,0,0,24,19,0,0,0,8,5,2,0,0,0,8,-1,0,-1,-1,-1,0,0,0,0,0,1$, $0,0,0,-1,-1,-1,-1,-1,0,0,0]$. From the viewpoint of Remark 3.2, we note that -496 is the degree of $\widetilde{L}(\Sigma)$ for the 2-local geometry $\Sigma$ of an $M_{12}$ in $\mathrm{C}(2 \mathrm{~B})$.

The Principal block. We compute $\widetilde{\Lambda}_{p r}^{C o_{3}}=[\overbrace{34263040}^{2^{12} \cdot 8365}, \frac{\mathbf{2 A}}{\mathbf{0}}, \frac{\mathbf{2 B}}{\mathbf{0}},-11840$, $544,-512,0,0,440,-40,0,0,0,0,0,-56,0,0,0,28,106,0,0,20,20,0$, $0,0,0,-40,44,0,0,0,-8,0,0,-37,-37,0,0,0]$, which is v-projective: $\widetilde{\Lambda}_{p r}^{C o_{3}}=\Phi\left(\varphi_{9}\right)+\Phi\left(\varphi_{10}\right)+6 \Phi\left(\varphi_{12}\right)+8 \Phi\left(\varphi_{14}\right)$.

Block 2-defect 3. $\widetilde{\Lambda}_{b 2}^{C o_{3}}=[\overbrace{13006720}^{2^{7} \cdot 101615}, \frac{\mathbf{2 A}}{\mathbf{0}}, \frac{\mathbf{2 B}}{-\mathbf{4 9 6}}, 11296,-2384,445,0,0$, $-80,155,0,0,0,8,5,34,0,0,0,4,-83,0,-1,-21,-21,0,0,0,0,16,-19$, $0,0,0,-17,-1,-1,36,36,0,0,0]$. This character fails either test. We can express this as: $\widetilde{\Lambda}_{b 2}^{C o_{3}}=4 \Phi\left(\varphi_{11}\right)+6 \Phi\left(\varphi_{13}\right)+11 \Phi\left(\varphi_{16}\right)+\chi_{32}+2 \chi_{38}$.

Block 3-defect 1. $\widetilde{\Lambda}_{b 3}^{C o_{3}}=[\overbrace{3108864}^{2^{12} \cdot 759}, \frac{\mathbf{2 A}}{\mathbf{0}}, \frac{\mathbf{2 B}}{\mathbf{0}},-1536,1056,192,0,0,-336$, $-96,0,0,0,0,0,24,0,0,0,-24,-24,0,0,0,0,0,0,0,0,24,-24,0,0,0,24$, $0,0,0,0,0,0,0]$. We can express this as $\widetilde{\Lambda}_{b 3}^{C o_{3}}=12 \Phi\left(\varphi_{15}\right)$. 
Theorem 8.1. $\widetilde{\Lambda}_{p r}^{C o 3}=\Phi\left(\varphi_{9}\right)+\Phi\left(\varphi_{10}\right)+6 \Phi\left(\varphi_{12}\right)+8 \Phi\left(\varphi_{14}\right)$, making $C_{3}$ a Class II "Principal Block Part V-Projective" group.

\section{The Rudvalis group}

The relevant maximal 2-local subgroups are $\left[\mathrm{CCN}^{+} 85\right.$, p. 126]:

$$
\begin{aligned}
& H_{1} \cong 2 \cdot 2^{4+6}: S_{5}, \\
& H_{3} \cong 2^{3+8}: L_{3}(2), \text { and } \\
& H_{6} \cong\left(2^{6}: U_{3}(3)\right): 2 \cong 2^{6} G_{2}(2) . \text { The diagram is: }
\end{aligned}
$$

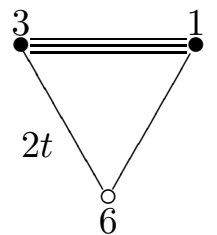

in the notation of [RS80, p. 288]. The diagram gives us the needed intersections, of the following structures:

$$
H_{16}=\frac{\frac{S_{3}}{2^{2+1+2}}}{2^{6}}, H_{36}=\frac{\frac{S_{3}}{2^{1+4}}}{2^{6}}, H_{136}=\frac{\frac{2}{2^{1+4}}}{2^{6}} \text {, and } H_{13}=\frac{\frac{S_{3}}{2^{2}}}{2^{3+8}} .
$$

These subgroups allow us to calculate:

$$
\widetilde{\Lambda}_{R u}=[32 a b]_{H_{6}} \uparrow^{R u}-[6 a]_{H_{3}} \uparrow^{R u}+[1 a]_{H_{1}} \uparrow^{R u}-1 .
$$

Indexed by conjugacy classes, we obtain: $\widetilde{\Lambda}_{R u}=[\overbrace{10113024}^{2^{12} \cdot 2469}, \frac{\mathbf{2 A}}{\mathbf{0}}, \frac{\mathbf{2 B}}{\mathbf{6 4}},-96,0$, $0,0,0,24,-1,0,5,0,0,0,0,-1,0,0,-1,1,1,1,-1,0,0,0,0,0,0,0,-1,-1$, $-1,-1,-1]$. This character does not vanish at the $2 \mathrm{~B}$ element, telling us that the Lefschetz module is non-projective. From the perspective of Remark 3.2, we see that 64 matches the degree of $\widetilde{L}(\Sigma)$, where $\Sigma$ is the building of $S z(8)$ in $\mathrm{C}(2 \mathrm{~B})$.

Block decomposition. $\widetilde{\Lambda}_{p r}^{R u}=[\overbrace{6881280}^{2^{16} \cdot 105}, \frac{\mathbf{2 A}}{\mathbf{0}}, \frac{\mathbf{2 B}}{\mathbf{0}},-48,0,0,0,0,280,80$, $0,28,0,0,0,0,0,0,0,-36,0,0,0,2,0,0,0,0,0,0,0,0,0,0,-14,-14$ ], which is v-projective: $\widetilde{\Lambda}_{p r}^{R u}=\Phi\left(\varphi_{5}\right)+\Phi\left(\varphi_{8}\right)$. Thus $R u$ belongs in Class II.

For block 2 (of defect 2), we find $\widetilde{\Lambda}_{b 2}^{R u}=[\overbrace{3231744}^{2^{12} \cdot 789}, \frac{\mathbf{2 A}}{\mathbf{0}}, \frac{\mathbf{2 B}}{\mathbf{6 4}},-48,0,0,0,0$, $-256,-81,0,-23,0,0,0,0,-1,0,0,35,1,1,1,-3,0,0,0,0,0,0,0,-1,-1,-1$, $13,13]$. This does not vanish at element $2 \mathrm{~B}$, so $\widetilde{\Lambda}_{b 2}^{R u}$ is non-projective. We minimize the number of non-projective characters and express the Lefschetz character of this block part as $\widetilde{\Lambda}_{b 2}^{R u}=\Phi\left(\varphi_{6}\right)+\Phi\left(\varphi_{7}\right)+6 \Phi\left(\varphi_{9}\right)+\chi_{36}$.

Theorem 9.1. $\widetilde{\Lambda}_{p r}^{R u}=\Phi\left(\varphi_{5}\right)+\Phi\left(\varphi_{8}\right)$. So Ru is a Class II "Principal Block Part V-Projective" group. 


\section{Summary}

We present a partition of the sporadic groups according to our findings, as described in Section 3 (boldface indicates groups originally classified by this work).

\begin{tabular}{|l|l|}
\hline Class & Sporadic Groups \\
\hline I: Lefschetz Module Projective & $M_{11}, J_{1}, M_{22}, M_{23}, J_{3}, M_{24}$, \\
& $M^{c} L, C o_{2}, L y, J_{4}, T h$ \\
\hline II: Principal Block Part V-Projective & $M_{12}, J_{2}, H S, \mathbf{S u z}, \mathbf{H e}, \mathbf{C o}_{\mathbf{3}}, \mathbf{R u}$ \\
\hline III: Principal Block Part Non-Projective & $\mathbf{O}^{\prime} \mathbf{N}$ \\
\hline
\end{tabular}

The groups $C o_{1}, F i_{22}, F i_{23}, F i_{24}^{\prime}, H N, B$, and $M$ have yet to be classified 8 The 2-modular decomposition matrices [Bre99] of these groups are not yet known. We do know by the $p$-test that their Lefschetz modules are non-projective, so they will be in Class II or Class III.

\section{Future DiRECTIONS}

For the block parts of Lefschetz modules that we found to have v-projective character, we suggest that the method of Steinberg module inversion by Webb Web87] as extended by Grodal Gro02 could possibly be used to show that the block part is actually a projective module in each case. The case of $O^{\prime} N$ should be investigated further; we found a number of unusual features (see Section [6) that made this group stand out. There are still seven sporadic groups whose Lefschetz characters could be computed (but they could not be fully decomposed mod 2 since the 2-modular irreducibles of these groups are not yet known). For even further directions, some different geometries for each group could be studied, as indicated by Benson-Smith [BS07]. The block decomposition corresponding to primes $p \neq 2$ could also be explored.

\section{REFERENCES}

[BCR90] D. J. Benson, J. F. Carlson, and G. R. Robinson, On the vanishing of group cohomology, J. Algebra 131 (1990), no. 1, 40-73. MR MR1054998 (91c:20073)

[Ben02] D. J. Benson, The nucleus and extensions between modules for a finite group, Representations of algebra. Vol. I, II, Beijing Norm. Univ. Press, Beijing, 2002, Easiest to find at http://www . maths.abdn.ac.uk/ bensondj/html/archive/benson.html, pp. 145-155. MR MR2067376 (2005c:20018)

[Bre99] Thomas Breuer, Decomposition Matrices, See details at http://www . math.rwth-aachen.de/ MOC/decomposition/, 1999.

[BS07] David J. Benson and Stephen D. Smith, Classifying spaces of sporadic groups, Surveys and Monographs of the AMS, American Mathematical Society, 2007, Submitted for publication. Preprint available at http://www . maths.abdn.ac.uk/ bensondj/html/archive/benson-smith.html

\footnotetext{
${ }^{8}$ Maginnis and Onofrei MO07] have recently classified $F i_{22}$ into Class II.
} 
[BW95] D. J. Benson and C. W. Wilkerson, Finite simple groups and Dickson invariants, Homotopy theory and its applications (Cocoyoc, 1993), Contemp. Math., vol. 188, Amer. Math. Soc., Providence, RI, 1995, pp. 39-50. MR MR1349127 (96d:55009)

$\left[\mathrm{CCN}^{+} 85\right]$ J.H. Conway, R.T. Curtis, S.P. Norton, R.A. Parker, and R.A. Wilson, Atlas of finite groups, Oxford University Press, 1985.

[CR94] Jon F. Carlson and Geoffrey R. Robinson, Varieties and modules with vanishing cohomology, Math. Proc. Cambridge Philos. Soc. 116 (1994), no. 2, 245-251. MR MR1281544 (95c:20073)

[Fei82] Walter Feit, The representation theory of finite groups, North-Holland Mathematical Library, vol. 25, North-Holland Publishing Co., Amsterdam, 1982. MR MR661045 (83g:20001)

[Gro99] The GAP Group, Gap — Groups, Algorithms, and Programming, Version 4.2, (http://www-gap.dcs.st-and.ac.uk/ gap) (Aachen, St. Andrews), 1999.

[Gro02] Jesper Grodal, Higher limits via subgroup complexes, Ann. of Math. (2) 155 (2002), no. 2, 405-457. MR MR1906592 (2003g:55025)

[MO07] John Maginnis and Silvia Onofrei, Fixed point sets of involutions, unpublished (2007), Preprint available at http://www.math.ksu.edu/ onofrei/fixedpoint.pdf

[RS80] M.A. Ronan and S. D. Smith, 2-local geometries for some sporadic groups, Proceedings of Symposia in Pure Mathematics 37 (1980), 283-289.

[RSY90] A. J. E. Ryba, Stephen D. Smith, and Satoshi Yoshiara, Some projective modules determined by sporadic geometries, Journal of Algebra 129 (1990), 279311 .

[Saw06] Masato Sawabe, On the reduced Lefschetz module and the centric p-radical subgroups. II, J. London Math. Soc. (2) 73 (2006), no. 1, 126-140. MR MR2197374 (2006j:20078)

[Smi90] Stephen D. Smith, On decomposition of modular representations from CohenMacaulay geometries, Journal of Algebra 131 (1990), 598-625.

[Smi05] Cohomology decomposition from subgroup complexes of finite groups,

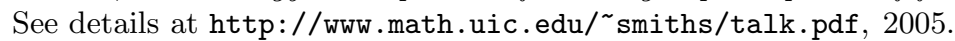

[Web87] P. J. Webb, A local method in group cohomology, Comment. Math. Helv. 62 (1987), no. 1, 135-167. MR MR882969 (88h:20065)

Department of Mathematics (M/C 249), University of Illinois at Chicago, 851 S. Morgan, Chicago, IL 60607, USA

E-mail address: grizzard@math.uic.edu 